\title{
MJN VENTILATOR ASSOCIATED PNEUMONIA: A SYSTEMATIC APPRAISAL IN A SAUDI ARABIAN CONTEXT
}

\author{
Catherine Jong ${ }^{1 *}$, Manfred Mortell ${ }^{2}$ \\ ${ }^{1}$ Medical Cardiac ICU, King Abdulaziz Cardiac Centre, King Abdulaziz Medical City Riyadh, Kingdom of \\ Saudi Arabia \\ ${ }^{2}$ Department of Nursing; Faculty of Medicine and Health Sciences University of Malaysia, Malaysia \\ *Corresponding Author's Email: catherinejongmt@gmail.com
}

\begin{abstract}
Background: Ventilator-associated pneumonia (VAP) is the commonest hospital-acquired infection (HAI) in intensive care. The incidence of VAP is the leading cause of HAI in the ICU. In the United States of America, VAP is the second most prevalent HAI with a reported mortality of $40-80 \%$. However, despite transparency in the literature on the preventative measures in the preceding decade, the prevalence of VAP remains high in mechanically ventilated patients and increases an ICU patient's confinement by 5-7 days once they developed a VAP. Methods: To determine the incidence, prevalence, and aetiology of VAP and oral care practices in Saudi Arabia, a systemic review of the literature published from the year 2000 till present day was employed. Databases searched included CINAHL, WILEY, PROQUEST and SCIENCE DIRECT. The systematic collection of data was followed by the development of the appraisal protocol which set out the methods in the appraisal This protocol specifies the appraisal question, inclusion and exclusion criteria, databases search strategies, study selection, quality appraisal and data production as well as the individual types of studies sited, appraised and synthesized. Results: The findings from the systematic appraisal indicate that implementing a comprehensive and effective oral care protocol which incorporates both the application of an antiseptic such as Chlorhexidine $0.12 \%$ and the clinical practice of tooth-brushing plays an instrumental role in preventing and reducing VAP in the intubated, mechanically ventilated ICU patient. Conclusions: VAP remains a common cause of HAI, but in order to stay current and compliant with knowledge and practical skills, ICU nurses require ongoing evidence-based education, reinforced with evidence-based practice. In addition to encouraging development of a body of knowledge that will advance nursing practice which is clinically safe and effective.
\end{abstract}

\section{Keywords: Middle East, Oral care; Saudi Arabia, Ventilator-associated pneumonia}

\section{INTRODUCTION}

"An ounce of prevention is worth a pound of care" (Benjamin Franklin). This quote, from an iconic and historic figure; is the personification of this systematic review.

Good health depends in part on a safe environment and practices that control or prevent the transmission of infections which help to protect patients from attaining diseases. Patients in an Intensive Care Unit (ICU) however, are at risk of acquiring Healthcare Associated Infections (HAI) because of their lowered immunity and exposure to invasive procedures (Stonecypher, 2010).
Patients in the ICU are therefore at risk of increased morbidity and mortality, not only from their admission illness but also from a HAI. A Healthcare Associated Pneumonia (HAP) is the most common HAI acquired in an ICU globally (Rosenthal et al., 2016; Stonecypher, 2010) and is the second most common HAI in critically ill patients (Graves, 2010). Curiously 86\% of HAP are related to mechanical ventilation and are labelled as Ventilator Associated Pneumonia (VAP) (Graves, 2010). A VAP continues as an evolving concept in critical care medicine and remains a substantial clinical predicament for ICU patients. The VAP however, is not a recent dilemma, and prevention strategies; education and 
research are an ongoing progress. Ventilator associated pneumonia is recognized as a common dilemma that occurs in mechanically ventilated patients. Patients who develop VAP have a higher mortality, increased delays in their recovery; endure prolonged hospital confinement, escalating healthcare expenses; and yet, this HAP is preventable (Melsen, et al., 2013; Alp et al., 2012; Stonecypher, 2010). Graves (2010) emphasizes that prevention of VAP is possibly one of the ultimate costeffective interventions currently achievable in the ICU. The objective of this systematic appraisal of the literature in a nursing context, is to inform healthcare professionals (HCP) concerning the incidence of VAP, prevention strategies, intervention guidelines and the importance of further research.

\section{Background}

The incidence of VAP is the leading cause of HAI in the ICU (Sedwick et al., 2012). In the United States of America (USA), VAP is the second most prevalent HAI with a reported mortality of $40-80 \%$ (Stonecypher, 2010). However, despite transparency in the literature on the preventative measures in the preceding decade, the prevalence of VAP remains as high as $23 \%$ in mechanically ventilated patients (Dellit, Owens \& McGowens, 2007). Augustyn (2007) declared that an ICU patient's length of stay is lengthened by 5-7 days once they developed a VAP. This increased length of stay converts to an increase in hospital expenses and in the USA, a diagnosis of VAP augments the cost of management on average by $\$ 20,000$ (Restrepo et al., 2010 ) to $\$ 40,000$ in a solitary admission (Safdar et al., 2005). Globally the prevalence of VAP spans 13-51 cases/1000 ventilator days (Joseph et al., 2010); however, the incidence of VAP differs internationally. The incidence of VAP cases per 1000 ventilator days in Saudi Arabia is 15.9 (Al-Dorzi et al., 2012), Brazil 10.416.4 (Marra et al., 2009), Canada 10.6 (Muscedere Martin \& Heyland, 2008) and in Kuwait 9.1(Aly et al., 2008). The National Healthcare Safety Network in 2011 reported 3,525 cases of VAP, equating to $0.0-4.9$ cases/1,000 ventilator days (Sedwick et al., 2012).

Given the previous statistics, it would be judicious to deduce that the incidence, cost of treatment and the alarming mortality rates associated with VAP are indicative of either a knowledge deficit pertaining to VAP or a failure to translate knowledge about VAP into clinical practice (Vandijck et al., 2010). Contemporary healthcare practices and delivery of care abide by the evidence-based practice (EBP) paradigm, the goal to provide safe, high quality healthcare with a goal to improve patient outcomes (Sedwick et al., 2012). It is therefore an issue of paramount importance that nurses remain current with EBP in order to advance clinical practices and subsequently patient care. The Institute for Healthcare Improvement (IHI) acknowledged the value of nursing interventions such as effective oral care (IHI, 2013). Alarmingly, despite the Joint Commission International (JCI) sanctions to employ the incidence of VAP statistics as a nursing-sensitive performance indicator in 2009 and listed it as a national patient safety goal effective January 2013 to reduce the incidence of the VAP (JCI, 2013). VAP remains a complex problem for ICU nurses and their ventilated patients in the ICU. The ICU nurses' role is therefore integral to the prevention and treatment of VAP in the mechanically ventilated patient(IHI, 2013; JCI, 2013).

Traditionally, oral care in the ICU has concentrated on patient comfort instead of plaque elimination, however in a technologically oriented ICU; oral care is regarded as of low importance (Yeung \& Chui, 2010).

Consequently, nursing research involving oral care and VAP prevention is essential to standardize best practice in the ICU setting. Can nursing actions decrease the incidence of VAP? That remains an empirical question; however, further research could provide crucial data to provide effective strategies. Currently in the literature, there is no standard, consistent or reliable methodology for the provision of oral care for intubated patients receiving mechanical ventilation in the ICU (Berry et al., 2011). There are however, studies that address numerous procedures for oral care in allinclusive ICUs (Cutler \& Sluman, 2014; Stonecypher, 2010; Yeung \& Chui, 2010); nonetheless, there is limited data existing for oral care in the mechanically ventilated patient admitted to the ICU in Saudi Arabia. Hence, an oral care protocol for intubated patients receiving mechanical ventilation in the ICU is consequently essential to establish best practice in order to prevent or reduce the incidence of VAP. Consequently, in order to transform clinical practice and reprioritize the importance of oral care in the ICU, EBP oral care strategies are required and mandatory.

This systematic appraisal first, describes the methods used for the provision of oral care in the 
intubated, mechanically ventilated ICU patient in Saudi Arabia; second, the corresponding frequency of oral care implementation and third, the intensive care nurse's mind-set towards delivering oral care. The conclusion of this systematic appraisal will be employed as baseline data to develop guidelines for providing oral care for intubated patients receiving mechanical ventilation in a Saudi Arabian ICU.

Nurses' employed in adult ICU in Saudi Arabia are multi-national, have a diverse educational background, in addition to numerous and varied clinical practices. Therefore, the clinical practice and comprehension of oral care practices are not standardized mind-set and varies from nurse to nurse.

Despite the relative simplicity, oral care practices are seen as a low priority nursing care intervention that can be disregarded; rather than an essential tool for effective infection prevention and control method. In addition, disturbingly, when nurses give oral care, it is often done erroneously, sourced from out-of-date information and habits instead of on EBP (Grap et al., 2003). As a clinical resource nurse employed in an adult medical ICU, I was determined to develop a comprehensive protocol for the delivery of oral care for intubated ICU patient's receiving mechanical ventilation. In doing so, my objective was to improve patient safety, the quality of patient care and consequently infection prevention and control practices in the ICU.

\section{METHODOLOGY}

The systematic collection of data followed the development of the appraisal protocol which set out the methods in the appraisal (Table 1). This protocol specifies the appraisal question, inclusion and exclusion criteria, databases search strategies, study selection, quality appraisal and data production as well as the individual types of studies sited, appraised and synthesized. The appraisal protocol determined the search method which was employed and included all the relevant studies which reduced the risk of introducing bias (CRD, 2009). The appraisal protocol described the methods being reviewed, the comprehensiveness of the review, the extent to which the review is replicable and quality of the final product (Bronson \& Davis, 2012).

The research question was established to accomplish the conclusion for the study; therefore, a conceptualization of the oral care bundles to prevent
VAP required keywords related to the chosen area of interest in conjunction with the central focus, the research study's aims and questions. Concluding with vital points of relevance, for the reader's interest and learning requirements (Punch, 2006). The researchers also affirmed that the inclusion-exclusion criteria used identified whether the studies chosen were relevant for the systematic review. The inclusion-exclusion criteria illustrate the studies chosen, the interventions employed, the comparison made, outcomes defined, the context of the studies and concluded with the types of reports that were included and those to be excluded. It was also noteworthy that transparency was important for researchers when determining which studies met the criteria to reduce bias when selecting articles for the systematic review (Bronson \& Davis, 2012). Since the systematic review should address and answer questions relating to strategies that can prevent VAP, it was encouraged to rely on experimental and quasiexperimental studies (Petticrew \& Roberts, 2006). Once the protocol document and inclusion-exclusion criteria were finalized, the authors explored the literature for relevant studies by reviewing titles and abstracts. When the articles were considered to be relevant, they were examined using established inclusion criteria and were subsequently critically re-appraised before being accepted as part of the systematic review.

Table 1: Appraisal Protocol Document (Bronson \& Davis, 2012)

\begin{tabular}{|c|c|}
\hline Study Table & The Effect of Oral Care Bundles on Prevention of VAP \\
\hline Research Aim & $\begin{array}{l}\text { The aims of the study are to establish whether implementing } \\
\text { oral care bundles can reduce VAP in intubated, mechanically } \\
\text { ventilated ICU patients compared to current practices. }\end{array}$ \\
\hline $\begin{array}{l}\text { Specific aims of } \\
\text { the study }\end{array}$ & $\begin{array}{l}\text { 1. To verify current oral care practices provided by nurses } \\
\text { in patients receiving mechanical ventilation } \\
\text { 2. To establish best practice oral care bundles } \\
\text { 3. To determine the oral care compliance rate of ICU } \\
\text { nurses following a standardized education program }\end{array}$ \\
\hline $\begin{array}{l}\text { Research } \\
\text { Questions }\end{array}$ & $\begin{array}{l}\text { 1. What are the possible causes of VAP in intubated, } \\
\text { mechanically ventilated patient? } \\
\text { 2. What does the evidence say about strategies to prevent } \\
\text { the risk of VAP in mechanically ventilated, ICU } \\
\text { patients? } \\
\text { 3. Does an implemented oral care bundle provide } \\
\text { outcomes that reduce VAP in intubated, mechanically } \\
\text { ventilated ICU patients? }\end{array}$ \\
\hline Scope & $\begin{array}{l}\text { The appraisal seeks to examine the causes of VAP in intubated, } \\
\text { mechanically ventilated ICU patients. It further investigates oral } \\
\text { care bundles strategies to reduce the incidences of VAP in } \\
\text { intubated, mechanically ventilated, ICU patients }\end{array}$ \\
\hline
\end{tabular}




\section{Establishing the Search Strategy}

The PICOC framework (Petticrew \& Roberts, 2006) was utilized and applied as a search strategy and the search terms were developed as follows in Table 2: $\mathrm{P}$ - patient; I - intervention; $\mathrm{C}$-comparison; $\mathrm{O}$ - outcome; $\mathrm{C}$ - context

Table 2: Application of PICOC Model (Petticrew \& Roberts, 2006)

\begin{tabular}{|l|l|l|l|}
\hline No & Term & Details & Rationale \\
\hline 1. & Patient & Mechanically ventilated patients & $\begin{array}{l}\text { This is the particular } \\
\text { group under study }\end{array}$ \\
\hline 2. & Intervention & Oral care bundle & $\begin{array}{l}\text { Oral care bundle: Are } \\
\text { strategies to reduce the } \\
\text { risk of ventilator- } \\
\text { associated pneumonia }\end{array}$ \\
\hline 3. & Comparison & Current oral care practices & $\begin{array}{l}\text { Routine oral care } \\
\text { practices by nurses }\end{array}$ \\
\hline 4. & Outcome & $\begin{array}{l}\text { Incidence of ventilator associated } \\
\text { pneumonia }\end{array}$ & $\begin{array}{l}\text { Oral care bundle: Are } \\
\text { effective to reduce the } \\
\text { risk of ventilator- } \\
\text { associated pneumonia }\end{array}$ \\
\hline 5. & Context & Intensive care unit & $\begin{array}{l}\text { Patient intubated and } \\
\text { mechanically ventilated } \\
\text { were admitted to high } \\
\text { acute area }\end{array}$ \\
\hline
\end{tabular}

\section{Conceptual Framework}

A useful scoping exercise that helped the researcher to focus on the topic identified concepts, dimensions and indicators for the prevention of VAP (Table 3). Utilizing the concept; that reducing oral bacterial colonization by employing oral care bundles; will promote the prevention of a VAP.

\section{Table 3: Scoping Exercise}

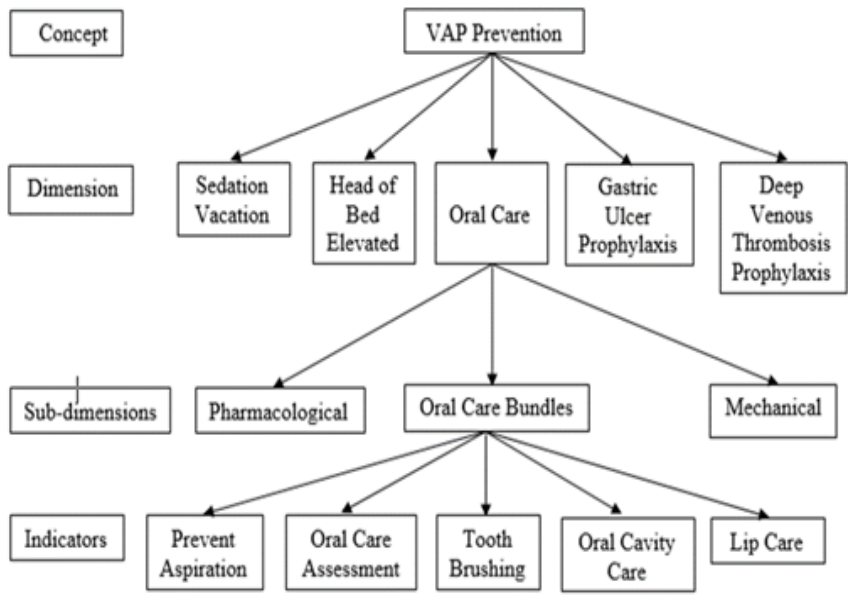

\section{RESULT}

The inclusion criteria for the literature review for this study, was to be written in the English language; to be specifically about oral care bundles and VAP in Saudi Arabia; to be Quantitative or Experimental; to have been published from 2009 onwards and to be from a peer reviewed journal. There were no studies published from Saudi Arabia that were retrieved in the literature search. Though expected, but, not surprising, the result was still disappointing, which meant that the inclusion criteria had to be widened and extended to other countries. The revised inclusion criteria were therefore; published in the English language; explicitly oral care bundles and VAP, related to mechanical ventilated patients; published from 2000; Quantitative or Experimental; and from a peer reviewed study.

The 'keyword searching' was free-text and utilized Boolean operators; as it limited the database "hits" which made the search more effective (Bronson and Davis, 2012). In addition to these two strategies, the EPPICentre (2010) and the CRD (2009) provided guidelines that located additional relevant articles. The literature search was conducted using the databases, Cumulative Index for Nursing and Health literature (CINAHL), WILEY, PROQUEST and SCIENCE DIRECT. The key words used were Oral care AND Ventilator Associated Pneumonia AND Saudi Arabia.

The key words were applied without any limiters and the resultant lack of hits required the search terms to be widened to include, Middle East: with Oral care AND Ventilator Associated Pneumonia. The search results for primary studies from the 4 databases yielded a total of 2,286 "hits" (Table 4). A total of 2,256 studies were rejected subsequent to the initial screening of the title using the inclusion criteria. These resulted in the screening and recognition of duplicated and repetitive studies. Abstracts of 32 potentially relevant studies were examined and 12 of these studies were excluded. This formed the first stage of the appraisal where a total of 20 studies were chosen as suitable for the appraisal (Table 5).

\section{Table 4: Search Results}

\begin{tabular}{|c|c|c|}
\hline No & Database & Number of Hits \\
\hline 1 & CINAHL & 1249 \\
\hline 2 & WILEY & 532 \\
\hline 3 & PROQUEST & 174 \\
\hline 4 & SCIENCE DIRECT & 331 \\
\hline & Total & 2286 \\
\hline
\end{tabular}


Table 5: Summary of Literature Search and Study Selection

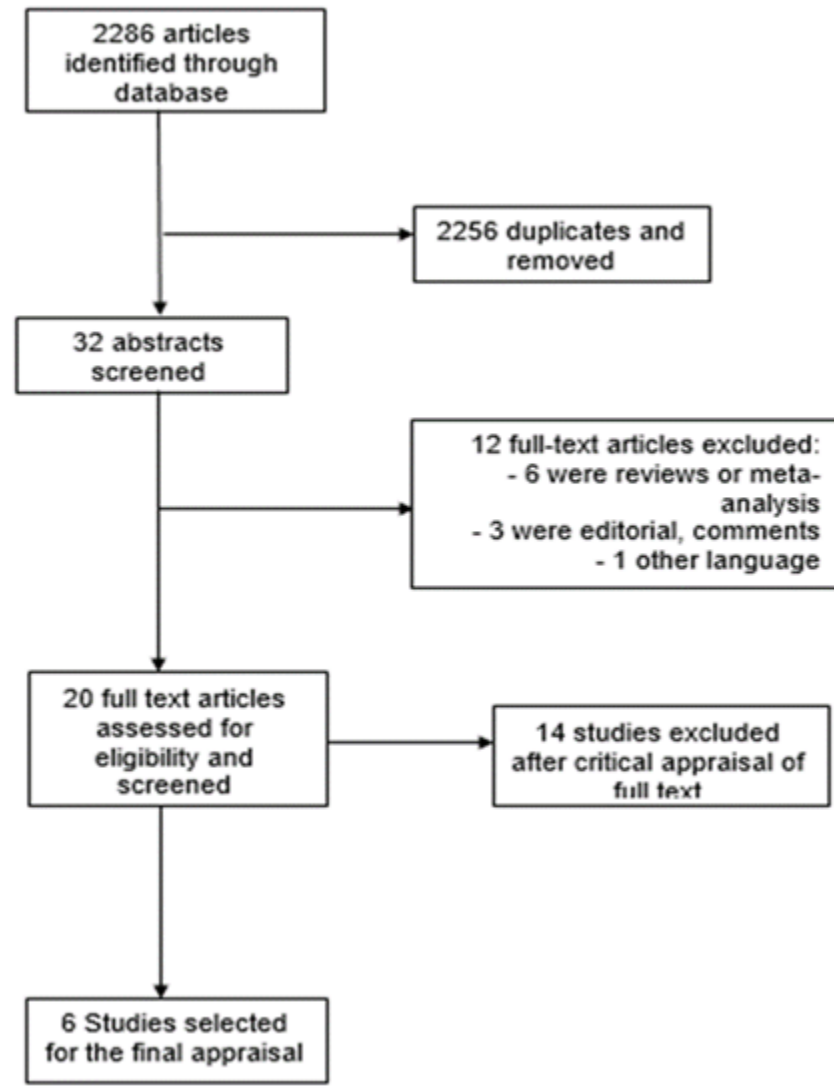

\section{Quality Assurance and Data Analysis}

For the purpose of this systematic appraisal, the 20 articles which met the inclusion criteria were reviewed for the appraisal. Throughout the process, the researcher noted the studies that made use of keywords as they provided the foundation for the analysis. This stage involved critical appraisal and weighting of the inclusion criteria. The activity associated with the quality of the appraisal involves identifying strengths and weaknesses in research articles, which evaluates and re-evaluates the importance of both. It is the critical appraisal stage that confirms whether the chosen 20 articles should be included in the systematic appraisal.

During this stage, relevant information from each study was recorded onto the critical appraisal skills program (CASP, 2010) checklist and critically appraised to determine the appropriateness for the study abstract, study method, participation, intervention, results, ethical considerations and conclusion.

Using elements from the CASP checklist for critiquing quantitative research (Glasper \& Rees, 2013); a seven scoring items quality assessment checklist was utilized for assessing the quality of the studies used for this appraisal. The scoring approach permitted assessment of whether or not a study met the criteria (2 points), partially (1 point) or not at all ( 0 point). A maximum score of 14 points for a research study was achievable.

Table 6 displays the quality evaluation for the reviewed studies.

Table 6: Individual Article Scoring Sheet (CASP, 2010)

\begin{tabular}{|c|c|c|c|c|}
\hline \multicolumn{5}{|c|}{ Article Number: } \\
\hline \multicolumn{5}{|c|}{ Full Reference of the article: } \\
\hline No & Criteria & Met & $\begin{array}{l}\text { Partially } \\
\text { Met }\end{array}$ & $\begin{array}{l}\text { Weighting } \\
\text { Score }\end{array}$ \\
\hline 1 & $\begin{array}{l}\text { Clear title and abstract with a } \\
\text { balanced summary of the whole } \\
\text { research }\end{array}$ & & & 2 \\
\hline 2 & $\begin{array}{l}\text { Participant is mechanically ventilated, } \\
\text { patient with an age of } 18 \text { years and } \\
\text { above }\end{array}$ & & & 2 \\
\hline 3 & $\begin{array}{l}\text { Methods: study design, study } \\
\text { population, eligibility criteria and data } \\
\text { collection and reliability of data } \\
\text { collection tools clearly stated }\end{array}$ & & & 2 \\
\hline 4 & $\begin{array}{l}\text { Interventions: clearly defined areas of } \\
\text { interest data measurements, statistical } \\
\text { methods and reliability and validity of } \\
\text { methods }\end{array}$ & & & 2 \\
\hline 5 & $\begin{array}{l}\text { Results: descriptive data, outcome } \\
\text { data and main results clearly stated }\end{array}$ & & & 2 \\
\hline 6 & $\begin{array}{l}\text { Ethical approval or informed consent } \\
\text { clearly stated }\end{array}$ & & & 2 \\
\hline 7 & $\begin{array}{l}\text { Discussion: key results discussed } \\
\text { relating to objectives and wider } \\
\text { evidence }\end{array}$ & & & 2 \\
\hline \multicolumn{4}{|c|}{ Total Score } & 14 \\
\hline
\end{tabular}

\section{Ethical Considerations}

The ethics of a systematic appraisal as a form of systematic research was adhered to by the researcher. Ethics for this study required the elimination of researcher bias in the selection methodology of the studies; which contributed to the study's transparency, and increased reliability and reproducibility (Gerrish et al., 2008). The researcher acknowledged that research data is an integral requirement for academic trustworthiness, and reliability which must also be considered for a systematic review. Therefore, this systematic appraisal adhered to the guidelines provided by the Research Ethics Committee (2011) of the University of Northumbria, UK in collaboration with the regulations for educational research endorsed by the British Educational Research Association (BERA, 2011). 


\section{DISCUSSION}

Oral care is a fundamental aspect of patient care in the mechanically ventilated patient and ICU nurses often trivialize its importance, in relation to clinical practice. To compound the issue, there are limited oral care protocols to guide nursing practice and as a consequence, VAP have a significant impact on patient morbidity and mortality. Despite the reality that oral care is a routine procedure in the ICU; the frequency and type of oral care practices vary amongst critical care nurses. The diverse oral care practices in the ICU; indicates the necessity for a standardized oral care protocol which includes both tooth-brushing and the use of a Chlorhexidine mouthwash (Berry et al., 2011). Furthermore, ensuring that a Chlorhexidine mouthwash and toothbrushes are available in the ICU stock may assist in implementing these EBP and consequently improve patients' outcomes in relation to the acquisition of a VAP.

This systematic appraisal has revealed important insights into present-day nurses' oral care practices and their attitudes toward implementing them for their patients. In doing so, the systematic appraisal has identified matters that not only need to be addressed with essential evidence-based research; but also, the future advancement of evidence-based oral care practices that nurses provide in hospitals.

Oral care remains a basic, yet integral patient care procedure that ICU nurses provide for their patients. The challenge is to promote compliance in a setting where the delivery of oral care to the intubated, mechanically ventilated ICU patient, is often dismissed due to the 'high-tech' environment in the ICU (Vandijck et al., 2010). Nevertheless, oral care must not be ignored in this 'high-tech' setting because ineffective oral health can result in a VAP, when bacteria-laden secretions which should have been sanitized migrate from the oropharynx into the lung. Therefore, effective oral care remains a fundamental nursing intervention for the intubated patient receiving mechanical ventilation; the rationale is because saliva production in these patients' decreases and chewing is impractical. As a consequence of the tracheal intubation; the growth of dental biofilm proliferates and a reservoir for destructive pathogens develops which may trigger the beginning of a VAP (Klompas, 2010).

The literature informs nurses that EBP guidelines are available to reduce the incidence of acquiring a VAP; the concern, however, is that these evidence-based recommendations have been accessible for several years, but unfortunately, EBP suggestions do not always bring about positive changes in behavior or in clinical practice. The literature seems to imply that healthcare practitioners are not incorporating best practice evidence into their patient care (Reinhardt, 2010). Which suggests that there is evidence and a concern regarding a theory practice gap. A study by Berry (2013) indicated that evidence-based oral care may prevent VAP, as it appears to reduce the number of destructive pathogens in the oral cavity and therefore validates the necessity for ICU nurses to provide effective oral care to their patients. This highlight yet another concern, which focuses on the fact that not all ICU nurses follow evidence-based oral care practices. Berry (2013) also suggested that oral care for intubated, mechanically ventilated ICU patient should include using a Chlorhexidine mouthwash, combined with gentle tooth-brushing, oropharynx suctioning and regular oral health assessments. Additional studies specifically validated the effectiveness of $0.12 \%$ Chlorhexidine gluconate as an antiseptic and antibacterial action against gram-positive and gram-negative organisms (IHI, 2013). However, despite the positive rhetoric about oral care recommendations, there are no consistent endorsements in the literature to determine what the most effective oral care practice is for intubated, mechanically ventilated ICU patients. The effects of tooth-brushing and use of a Chlorhexidine mouthwash have been used in combination to assess the benefits in preventing a VAP. The literature appraisal has revealed that although toothbrushing removed dental biofilm, if used in isolation, did not prevent the acquisition of a VAP. However, using an oral Chlorhexidine mouthwash as the sole intervention; did significantly reduce the incidence of VAP. Therefore, the combination of tooth- brushing and use of an oral Chlorhexidine mouthwash, revealed identical outcomes, when compared to Chlorhexidine mouthwash used without tooth-brushing. These results are therefore positive and suggest that VAP prevention is possible if the clinical practices include the use of an oral Chlorhexidine mouthwash.

The practice of tooth-brushing to maintain good oral health in the general population has been and will continue to be a standard practice. However, the effect of tooth-brushing in the ICU patient with mechanical ventilation remains indeterminate. In fact, researchers have cautioned; that using tooth-brushing maybe 
counterproductive as it could dislodge pathogens from the dental plaque which could translocate them into the lung and initiate a VAP (Pobo et al., 2009). Therefore, despite varied recommendations for reducing dental plaque and pathogen colonization in the oral cavity of the intubated, mechanically ventilated ICU patient, the most effective and safest method still remains unknown (Berry, 2013).

The critical analysis of the VAP related literature was challenging because the methodologies, interventions and protocols examined were diverse, and revealed a range of practices which made validation of individual praxes effectiveness awkward. Again, confirming that a gap exists not only in the literature regarding VAP, but also in clinical practices for the prevention or reduction of VAP. However, the 6 VAP studies reviewed did reveal that positive patient outcomes could be achieved with the use of Chlorhexidine antiseptics tooth- brushing and the removal of oral and pharyngeal secretions (Klompas, 2010). Of the 6 studies in the systematic appraisal, and oral care interventions, only 2 confirmed a significant decrease in the risk of acquiring a VAP by $33.33 \%$ and by $62.5 \%$. Therefore, again confirming disparity of evidence related to practice and outcomes for the prevention and reduction of VAP in the intubated, mechanically ventilated ICU patient.

The 4 themes that did emerge from the 6 studies for oral care and VAP prevention were, oral assessment, oral cavity care, preventing aspiration and preventing oral plaque formation.

1. Oral Assessment, mandated that the practitioner must first identify a potential or actual oral hygiene problem; and then provide the appropriate intervention with continued observation and oral assessment at least once per shift. The nursing oral assessment should focus on the normal and abnormal physical appearance of the lips, teeth, gingival, tongue, mucosa, saliva, soft and hard palate (Huskinson \& LIoyd, 2009).

2. Oral cavity care, required that the practitioner must be knowledgeable about the oral mucous as a potential source of anaerobic bacteria proliferation if associated with dry or cracked of oral tissue. The 6 studies strongly advocated maintaining the integrity of the oral mucosa, and thus the nurse must gently cleanse the oropharynx with a toothbrush or swab immersed with Chlorhexidine gluconate $0.2 \%$. The tracheal tube may also cause the lips to become dry and crack due to a patient's inability to moisten their lips, whether due to sedation, a tracheal tube or associated pressure from securing devices which immobilize the tracheal tube (Prendergast et al., 2012). Therefore, it is imperative to avoid pressure on the lips and apply an aqueous oral moisturizer once a shift for comfort and to minimize sites that could harbor bacteria (Feider, Mitchell \& Bridges, 2010).

3. Preventing aspiration of oral and pharyngeal secretions into the lung could be reduced by positioning the patient in a semi-recumbent position with head elevated to approximately $45^{\circ}$ (Prendergast et al., 2012; Klompas, 2010); in addition to measuring tracheal tube cuff pressure and maintaining a pressure range between 20-25mmHg (Pobo et al., 2009) to prevent the migration of oral secretions into the lung. And for the completeness of effective oral care, suctioning should be performed routinely (Prendergast et al., 2012).

4. Preventing oral plaque formation in the literature advocated tooth-brushing as a preventative intervention to remove plaque and limit its formation (Ames, 2011), in addition to reducing micro-organisms proliferation in the oral cavity (Halm \& Armola, 2009), which dissuades tongue biofilm development and halitosis (Prendergast et al., 2012). Tongue cleansing has also been recommended as an important intervention as it physically removes pathogens reducing their excess (Halm \& Armola, 2009).

In addendum to tooth-brushing and tongue cleansing, mouth rinsing with Chlorhexidine gluconate $2 \%$ has previously been recommended, however, Ames (2011) cautions that it is inadvisable to supplement the antiseptic with tap water for rinsing as it can be a source of HAI. Although oral care has been verified as essential to prevent VAP, evidence-based nursing protocols to impact with this HAI in the ICU are scarce. In defence of the oral care diversity and its implementation in clinical practice, there is minimal evidence-based knowledge about the benefits or effects of the oral care interventions for a mechanically ventilated and intubated patient. More of a concern however, is that the implementation of oral care protocols in clinical practice is still, amazingly, controversial (Hsu et al., 2010). It was interesting to note that all the studies that were analysed in the systematic appraisal demonstrated no agreement or consistency concerning a standardized oral care practice or the method for oral health, its application and the product selection. 
However, overall, Chlorhexidine was considered the antiseptic of choice as it demonstrated a reduction in incidence of VAP when compared to oral care without an antiseptic. The reported inconsistencies however included no data for the recommended frequency in the provision of oral care and the data pertaining to the effects of tooth-brushing on the incidence of VAP was inconclusive.

\section{Summary of the Systematic Appraisal}

Developing or adopting an evidence-based oral care protocol offers nurses and the nursing profession a fundamental practice which can not only reduce the incidence of VAP but also enhance patient safety from one of the most common and lethal HAI. The findings from the 6 studies appraised indicate that implementing a comprehensive and effective oral care protocol which incorporates both the application of an antiseptic such as Chlorhexidine $0.12 \%$ and the clinical practice of toothbrushing plays an instrumental role in preventing and reducing VAP in the intubated, mechanically ventilated ICU patient. VAP are a serious HAI, which has a higher morbidity, mortality, length of stay and healthcare economic cost than the intubated, mechanically ventilated patient who does not develop a VAP. VAP have gained notoriety and are infamous as the second most common HAI, a statement which is endorsed by the plethora of research that has been undertaken in an effort to gain insight into the best practices to prevent this deadly disease.

The 6 research studies reviewed in this paper indicate that oral care bundles are most consistent with markedly reduced VAP rates when nursing care practices consist of frequent interventions such as oral assessment, timed tooth-brushing, oral and pharyngeal suctioning, and oral cavity rinsing and moisturizing. To confirm these oral care practices are valid and effective, they have been acknowledged and recommended by numerous respected healthcare and accrediting organizations (IHI, 2013; CDC, 2013; JCI, 2013) as important clinical interventions that decrease the prevalence of VAP. Regrettably, there seems to be lack of consensus amongst these same healthcare and accrediting associations as to the best method of oral care that should be utilized by nurses caring for the intubated, mechanically ventilated ICU patient. However, despite the controversy and lack of cohesiveness amongst experts in clinical practice, there is generic acknowledgement that VAP are preventable and nursing as a professional body has made great strides towards combating VAP by implementing oral care bundles as an intervention. The absence however of EBP guidelines to standardize clinical practices, ratifies the ICU nurse to perform oral care according to individual preferences and historical nursing patterns. The creation, development and evidence-based validation of oral care protocols to reduce or prevent the incidence of VAP as a HAI is therefore not only essential, but also a mandate for patient safety and advocacy.

In response to the continued prevalence of the VAP (Al-Dorzi et al., 2012) and despite the implementation of ventilator bundles, oral care bundles have been a consequential recommendation from the evidence presented in this article. The oral care bundles proposed in this systematic appraisal should be used in conjunction with other elements of a preventative VAP bundle discussed. In addition to providing oral care, oral assessments should also be provided twice daily for all the intubated, mechanically ventilated ICU patients. Assessment and evaluation of the patients' oral history and actual physical assessment findings may provide information regarding a potential problem. Oral physical indicators such as gingivitis or periodontitis, increase the risk and prevalence of bacteraemia in the oral cavity (Ames, 2011). This assessment is not only a valuable tool that may identify whether a patient is at risk of developing a VAP, but, is also part of a normal nursing physical assessment which is performed twice daily or when there is a change in the patients' condition.

Guidelines for the reduction or prevention of a VAP by means of oral care bundles include, subglottic suctioning, removing oral plaque and associated microbe pathogens with previously stated toothbrushing and antiseptic mouthwash interventions (Table 6). Having oral care protocols in clinical practice was reported to increase the oral care quality and decrease the incidence of acquiring a VAP. Neglecting some or all of these interventions increase the risk for acquiring a VAP. The unacceptable high morbidity, mortality, and economic costs of treating patients with a VAP remains a concern not only for the profession of nursing, but for all healthcare professionals providing care in the ICU and to the intubated, mechanically ventilated patient. This means that even small gains in VAP prevention can translate into thousands of lives, decreasing morbidity and mortality figures and millions of dollars saved in additional healthcare costs. 


\section{Table 6: Oral Care Bundles}

\begin{tabular}{|c|c|}
\hline Steps & Oral care interventions \\
\hline \multirow[t]{2}{*}{1} & $\begin{array}{l}\text { Place the patient in a semi-recumbent position at a } 45^{\circ} \\
\text { angle }\end{array}$ \\
\hline & Maintain cuff pressure between $20-25 \mathrm{mmHg}$ \\
\hline 3 & $\begin{array}{l}\text { Oropharynx suction or deep suction of pooled secretions } \\
\text { in oropharynx pre and post oral care }\end{array}$ \\
\hline 4 & $\begin{array}{l}\text { Oral assessment on the lips, teeth, gingival, tongue, } \\
\text { mucosa, saliva, soft and hard palate performed twice } \\
\text { daily ( } 8 \mathrm{am}, 8 \mathrm{pm})\end{array}$ \\
\hline 5 & $\begin{array}{l}\text { Tooth-brushing using a soft bristled baby toothbrush } \\
\text { with a small amount of toothpaste every } 12 \text { hours ( } 8 \mathrm{am} \text {, } \\
8 \mathrm{pm} \text { ) }\end{array}$ \\
\hline 6 & $\begin{array}{l}\text { Tongue cleansing: place the toothbrush with toothpaste } \\
\text { gently and flush the surface of the tongue every } 12 \\
\text { hourly }(8 \mathrm{am}, 8 \mathrm{pm})\end{array}$ \\
\hline 7 & $\begin{array}{l}\text { Oral cavity care: swabbing the oral mucosa, gingival, } \\
\text { soft and hard palate with Chlorhexidine } 0.12 \% \text { every } 4 \\
\text { hours }\end{array}$ \\
\hline 8 & Mouth rinses with Chlorhexidine $0.12 \%$ every 4 hours \\
\hline 9 & $\begin{array}{l}\text { Lip care: apply aqueous oral moisturizer after oral care } \\
\text { twice daily }\end{array}$ \\
\hline
\end{tabular}

\section{CONCLUSION}

In order to stay current and compliant with knowledge and practical skills, ICU nurses require ongoing evidence-based education, reinforced with EBP. One of the goals of research is to develop a body of knowledge that will advance nursing practice by delivering evidence-based clinically effective nursing care. For this objective to be met, the following recommendations for the implementation of evidencebased clinical practice are suggested by the researcher.

1. Continuing education in-services on VAP and related topics must be maintained in order for ICU nurses to improve and maintain knowledge on prevention of VAP (Korhan et al., 2013).

2. The orientation of new ICU nurses should include education on the pathophysiology of VAP, the risk factors and strategies for prevention (Jansson et al., 2013).

3. VAP education must be provided to all paramedical personnel who have direct contact with ventilated patients; this includes respiratory therapists and patient care technicians (Arroliga et al., 2012).

4. The ICU nurse educator and the infection prevention and control team should provide ongoing education related to VAP prevention and best practice measures (Ames, 2011).

5. Continued implementation and assessment of the effectiveness of currently established oral care bundle elements should be compulsory (Lawrence \& Fulbrook, 2011).

6. Ongoing monitoring of the oral care compliance rate provided by ICU nurses following a standardized educational program (Cuccio et al., 2012).

7. Monthly audits to monitor the incidence of VAP, with a 'public' visual display of results to demonstrate transparency and effectiveness as a nursing sensitive quality indicator (JCI, 2013).

8. The utilization of the Nelson et al., (2006) ICU nurses' prioritization scale to rate task importance for the prevention of VAP:

- $\mathrm{C}$-Critical; Prevent aspiration

- U-Urgent; Tooth-brushing and oral cavity care

- $\mathrm{R}$-Routine; Lip care

- E-Extra; Oral assessment

\section{Recommendations for Further Research}

Poor oral care in the intubated, mechanically ventilated, intensive care patient was highlighted as a precursor to acquiring a VAP, also a HAI (Cuccio et al., 2012). Oral care for the prevention of a VAP was revealed to be an effective care intervention in numerous studies (Cutler \& Sluman, 2014; Ames, 2011), however, no research studies to date have provided a definitive evidence-based study that utilizes a standard interval for oral care which has reduced the occurrence of a HAI such as a VAP. The urgency is then, that healthcare organizational policies and procedures pertaining to the prevention of the VAP, need to state and enforce the, who, how, when and why requirements for the provision of oral care in the intubated, mechanically ventilated ICU patient. In addition, further research must be endorsed and performed to show statistical evidence that reduced bacterial colonization in the oral cavity is grounded on the frequency of oral care provided by nurses. The paradox is that the nurses, who provide this basic care inconsistently and in a non-standardized manner, yet have the ability to develop hospital policies and convene on committees which could authorize the implementation of evidencebased protocols for oral care.

Additional research studies are required for the 
validation and recommendations on the diverse variety of potentially effective oral care products such as: standard toothbrushes, mechanical toothbrushes, and oral swabs. Subsequent research utilizing these tools for the prevention of VAP incidence, must be recommended as they may demonstrate differences in the effectiveness that one tool has over the other and improve patient outcomes. These future research studies should also include other variables such as: the different time related intervals that oral care is performed, in doing so, if valid and effective as EBP can be implemented in the ICU with intubated, mechanically ventilated patients.

In conclusion, as the author and researcher, I reemphasize that "Prevention is better than cure" (popular Dutch Proverb) and this article has summarized the findings and discussion from this systematic appraisal. In addition, has highlighted the implications of the findings for future practice and further research. Nurse lead oral care bundle strategies have been demonstrated to be effective in the intubated, mechanically ventilated intensive care patient and in the critical care setting, where prevention of a VAP can significantly impact on morbidity, mortality, healthcare economics and the patients' prospects.

\section{Conflict of Interests}

The authors declare that they have no conflict of interest.

\section{ACKNOWLEDGEMENT}

The authors are thankful to the institutional authority for completion of the work.

\section{REFERENCES}

Al-Dorzi, H.M., El-Saed, A., Rishu, A.H., Balkhy, H.H., Memish, Z.A. \& Arabi, Y.M. (2012). The results of a 6-year epidemiologic surveillance for ventilator-associated pneumonia at a tertiary care intensive care unit in Saudi Arabia. American Journal of Infection Control, 40(9), pp 794-799.

Alp, E., Kalin, G., Coskun, R., Sungur, M., Guven, M. \& Doganay, M. (2012). Economic burden of ventilator-associated pneumonia in a developing country. Journal of Hospital Infection, 81(2), pp 128-130.

Aly, N.Y.A., Al-Mousa, H.H. \& Al Asar, E.S.M. (2008). Nosocomial infections in a medical-surgical intensive care unit. Internal Journal of the Kuwait University, 17(5), pp 373-377.

Ames, N. J. (2011) 'Evidence to support tooth brushing in critically ill patients. American Journal of Critical Care, 20(3), pp 242-250.

Augustyn, B. (2007). Ventilator-associate pneumonia: Risk factors and prevention. Critical Care Nurse, 27(4), pp 32-39.

Arroliga, A.C., Pollard, C.L., Wilde, C.D., Pellizzari, S.J., Chebbo, A., Song, J., Ordner, J., Cormier, S. \& Meyer, T. (2012). Reduction in the incidence of ventilator-associated pneumonia: A multidisciplinary approach. Respiratory Care, 57(5), pp 688-696.

Franklin, B. Infection Prevention Quotes \& Sayings. Retrieved from: http://www.searchquotes.com/ search/Infection_Prevention/

Berry, A.M. (2013). A comparison of Listerine ${ }^{\circledR}$ and sodium bicarbonate oral cleansing solutions on dental plaque colonisation and incidence of ventilator associated pneumonia in mechanically ventilated patients: A randomised control trial. Intensive and Critical Care Nursing, 29(5), pp 275-281.

Berry, A.M., Davidson, P.M., Masters, J., Rolls, K. \& Ollerton, R. (2011). Effects of three approaches to standardized oral hygiene to reduce bacterial colonization and ventilator associated pneumonia in mechanically ventilated patients: a randomised control trial. International Journal of Nursing Studies, 48(6), pp 681-688.

British Educational Research Association (BERA). (2011). Ethical Guidelines for Educational Research. Retrieved from: http://www.bera.ac.uk 
Bronson, D.E. \& Davis, T.S. (2012). Finding and Evaluating Evidence: Systematic Review and Evidence-Based Practice. London: Oxford University Press.

Centers for Disease Control and Prevention (CDC). (2013). Protocol Clarifications: Ventilator-Associated Pneumonia (VAP) Event. Retrieved from: www.cdc.gov/nhsn/pdfs/pscmanual/9pscssicurrent.pdf

Centre for Reviews and Dissemination (CRD), University of York. (2009). Systematic Reviews: CRD's Guidance for Undertaking Reviews in Health Care. Retrieved from: http://www.york.ac.uk

Critical Appraisal Skills Programme (CASP). (2010). Retrieved from: http://www.casp-uk.net/

Cuccio, L., Paradis, H., Rivet, C. \& Lynch, J. (2012). An Evidence-Based oral care protocol to decrease ventilatorassociated pneumonia. Dimensions of Critical Care Nursing, 31(5), pp 301-308.

Cutler, L.R. \& Sluman, P. (2014). Reducing ventilator associated pneumonia in adult patients through high standards of oral care: A historical control study. Intensive and Critical Care Nursing, 30(2), pp 61-68.

Dellit, T.H., Owens, R.C. \& McGowens, J.E. (2007). Infectious Disease Society America and the Society for Healthcare Epidemiology of America guidelines for developing an institutional program to enhance antimicrobial. Clinical Infectious Disease, 44(2), pp 159-177.

Evidence for Policy and Practice Information and Co-ordinating Centre (EPPI-Centre). (2010). EPPI-Centre Methods for Conducting Systematic Reviews. Retrieved from: http://www.eppi.ioe.ac.uk

Feider, L.L., Mitchell, P. \& Bridges, E. (2010). Oral care practices for orally intubated critically ill adults. American Journal of Critical Care, 19(2), pp 175-183.

Gerrish, K., Ashworth, P., Lacey, A. \& Bailey, J. (2008). Developing evidence-based practice: experiences of senior and junior clinical nurses. Journal of Advanced Nursing, 62(1), pp 62-73.

Glasper, A. \& Rees, C. (2013). How to write your nursing dissertation. West Sussex, United Kingdom: John Wiley and Sons, Ltd.

Grap, M.J., Munro, C.L., Bryant, S. \& Ashtiani, B. (2003). Predictors of backrest elevation in critical care. Intensive Critical Care Nursing, 19(2), pp 68-74.

Graves, N. (2010). The effect of healthcare acquired infections on length of hospital stay and cost. Infection Control and Hospital Epidemiology, 28(3), pp 280-292.

Halm, M. \& Armola, R. (2009). Effect of oral care on bacterial colonization and ventilator-associated pneumonia. American Journal of Critical Care, 18(3), pp 275-278.

Hsu, A.P., Liao, A.S., Li, C.Y. \& Chiou, A.F. (2010). The effects of different oral care protocols on mucosa change in orally intubated patients from an intensive care unit. Journal of Clinical Nursing, 20(7-8), pp 1044-1053.

Huskinson, W. \& LIoyd, H. (2009). Oral health in hospitalized patients: assessment and hygiene. Nursing Standard, 23(36), pp 43-47.

Institute for Healthcare Improvement (IHI). (2013). How-to Guide: Prevent Ventilator-Associated Pneumonia. Retrieved from: http://www.ihi.org

Jansson, M., Ala-Kokko, T., Ylipalosaari, P., Syrjala, H. \& Kyngas, H. (2013). Critical care nurses' knowledge of adherence to and barriers towards evidence-based guidelines for the prevention of ventilator- associated pneumonia- $\mathrm{A}$ survey study. Intensive and Critical Care Nursing, 29(7), pp 216-227. 
Joint Commission International (JCI). (2013). International Patient Safety Goals (IPSG) 5: Reduce the risk of Healthcare associated infection. The Joint Commission Journal on Quality and Patient Safety, 38(12), pp 1-3.

Joseph, N.M., Sistla, S., Dutta, T.K., Badhe, A.S. \& Parija, S.C. (2010). Ventilator-associated pneumonia: A review. European Journal of Internal Medicine, 21(5), pp 360-368.

Klompas, M. (2010). Prevention of ventilator-associated pneumonia. Expert Review of Anti-Infective Therapy, 8(7), pp 791-800.

Korhan, E.A., Yont, G.H. Kilic, S.P. \& Uzelli, D. (2013). Knowledge levels of intensive care nurses on prevention of ventilator-associated pneumonia. British Association of Critical Care Nurses, 19(1), pp 26-33.

Lawrence, P. \& Fulbrook, P. (2011). The ventilator care bundle and its impact on Evidence-based guidelines for the prevention of ventilator-associated pneumonia: Results of a knowledge test among European intensive care nurses. Journal of Infection Control, 70(2), pp 180-185.

Marra, A.R., Rodrigues, R.G., Silva, C.V., Caserta, R.A., Paes, A.T., Moura, D.F. Jr, dos Santos, O.F., Edmond, M.B. \& Durão, M.S. (2009). Successful prevention of ventilator-associated pneumonia in an intensive care setting. American Journal of Infection Control, 37(8), pp 619-625.

Melsen, W.G., Rovers, M.M., Groenwold, R.H., Bergmans, D.C., Camus, C., Bauer, T.T., Hanisch, E.W., Klarin, B., Koeman, M., Krueger, W.A., Lacherade, J.C., Lorente, L., Memish, Z.A., Morrow, L.E., Nardi, G., van Nieuwenhoven, C.A., O'Keefe, G.E., Nakos, G., Scannapieco, F.A., Seguin, P., Staudinger, T., Topeli, A., Ferrer, M. \& Bonten, M.J. (2013). Attributable mortality of ventilator-associated pneumonia: a meta-analysis of individual patient data from randomised prevention studies. The Lancet Infectious Diseases, 13(8), pp 665-671.

Muscedere, J.G., Martin, C.M. \& Heyland, D.K. (2008). The impact of ventilator-associated pneumonia on the Canadian health care system. Journal of Critical Care, 23, pp 5-10.

Nelson, J.E., Puntillo, K.A., Pronovost, P.J., Walker, A.S., McAdam, Ilaoa, D. \& Penrod, J. (2012). In their own words: Patients and families define high-quality palliative care in the intensive care unit. Critical Care Medicine, 38(3), pp 808-818.

Petticrew, M. \& Roberts, H. (2006). Systematic Reviews in the Social Sciences: A Practical Guide. Australia: Blackwell Publishing.

Pobo, A., Lisboa, T., Rodriguez, A., Sole, R., Magret, M., Trefler, S., Gomez, F. \& Rello, J. (2009). A randomized trial of dental brushing for preventing ventilator-associated pneumonia. Chest, 136 (2), pp 433-439.

Prendergast, V., Jakobsson, U., Renvert, S. \& Hallberg, I.R. (2012). Effects of a standard versus comprehensive oral care protocol among intubated neuroscience ICU patients: Results of a randomized controlled trial. Journal of Neuroscience Nursing, 44(3), pp 134-145.

Punch, K.F. (2006). Developing Effective Research Proposals. $2^{\text {nd }}$ edition. London: Sage Publication Ltd.

Reinhardt, J.P. (2010). Research methods in Evidence-Based Practice: Understanding the Evidence. Journal of the American Society on Aging, 34(1), pp 36-42.

Research Ethic Committee. (2011). Ethics in Research- Policy Statement. Northumbria University. Retrieved from: https://www.northumbria.ac.uk/research/ethics-and-governance/-/media/corporate-website/documents/pdfs/ research/ethics-in-research-policy-statement.ashx

Restrepo, M.I., Mortensen, E.M., Rello, J., Brody, J. \& Anzueto, A. (2010). Late admission to the ICU in patients with community-acquired pneumonia is associated with higher mortality. Chest, 137(3), pp552-557. 
Rosenthal, R., McGranahan, N., Herrero, J., Taylor, B.S \& Swanton, C. (2016). deconstructSigs: delineating mutational processes in single tumors distinguishes DNA repair deficiencies and patterns of carcinoma evolution. Genome Biology, 17(31).

Safdar N., Dezfulian C., Collard H.R. \& Saint S. (2005). Clinical and economic consequences of ventilator-associated pneumonia: A systematic review. Critical Care Medicine, 33(10) pp 2184-2193.

Sedwick, M.B., Smith, M.L., Reeder, S.J. \& Nardi, J. (2012). Using Evidence-Based Practice to prevent ventilatorassociated pneumonia. Critical Care Nurse, 32(4), pp 41-50.

Stonecypher, K. (2010). Ventilator-associated pneumonia: The importance of oral care in intubated adults. Critical Care Nursing Quarterly, 33(4), pp 339-47.

Vandijck, D.M., Labeau, S.O., Vogelaers, D.P. \& Blot, S.I. (2010). Prevention of nosocomial infections in intensive care patients. Nursing in Critical Care, 15(5), pp 251-256.

Yeung, K.Y. \& Chui, Y.Y. (2010). An exploration of factors affecting Hong Kong ICU nurses in providing oral care. Journal of Clinical Nursing, 19(21-22), pp 3063-3072. 